
allemande

\title{
Le débat sur la responsabilité de la guerre à l'ombre de Versailles, 1919-1933
}

\section{Gerd Krumeich}

Traducteur : Valentine Meunier et Martine Sgard (relecture)

\section{OpenEdition}

\section{Journals}

Édition électronique

URL : https://journals.openedition.org/allemagne/2422

DOI : 10.4000/allemagne.2422

ISSN : 2605-7913

\section{Éditeur}

Société d'études allemandes

\section{Édition imprimée}

Date de publication : 31 décembre 2020

Pagination : 303-315

ISSN : 0035-0974

\section{Référence électronique}

Gerd Krumeich, "Le débat sur la responsabilité de la guerre à l'ombre de Versailles, 1919-1933 »,

Revue d'Allemagne et des pays de langue allemande [En ligne], 52-2 | 2020, mis en ligne le 31 décembre 2021, consulté le 20 février 2022. URL : http://journals.openedition.org/allemagne/2422 ; DOI : https:// doi.org/10.4000/allemagne.2422 


\section{Le débat sur la responsabilité de la guerre à l'ombre de Versailles, 1919-1933}

\section{- Gerd Krumeich *}

\section{1. État actuel de la recherche et problématique}

La recherche sur cette question n’a pratiquement pas évolué depuis le début des années 1990. Au cours de la décennie 1980-1990, un certain nombre de travaux ont été entrepris dans le sillage du débat soulevé par les thèses de Fritz Fischer ${ }^{(1)}$. Ceux d'Ulrich Heinemann et Wolfgang Jäger, notamment, ont posé des jalons durables ${ }^{(2)}$. On peut y ajouter, au début des années 1990, les recherches exemplaires que Michael Dreyer et Oliver Lembcke ont consacrées au débat sur les responsabilités allemandes dans le déclenchement de la guerre à l'époque de la défaite et des négociations de Versailles ${ }^{(3)}$.

Néanmoins, tous ces travaux anciens posent problème. Leurs auteurs, et c'est particulièrement vrai pour Geiss et Heinemann ainsi que pour Lembcke et Dreyer, sont en effet bien trop intimement convaincus que la responsabilité du déclenchement de la guerre en 1914 incombe entièrement, ou en majeure partie, à l'Allemagne. En conséquence, ils ne fournissent aucun éclairage sur les contestations de l'entre-deuxguerres, qui n'émanaient pas uniquement des franges nazies ou conservatrices de la

* Professeur émérite à l'Université Heinrich Heine de Düsseldorf.

1 Pour une introduction à la «controverse Fischer ", voir Édouard Husson, "Les historiens et la mémoire du passé nazi en République fédérale d'Allemagne», Matériaux pour l'histoire de notre temps, $\mathrm{n}^{\circ} 68$ (2002), p. 14-19. L'article détaillé de Wikipedia cite presque toute la littérature scientifique sur le sujet: de.wikipedia.org/wiki/Fischer-Kontroverse ou fr.wikipedia.org/wiki/Controverse_Fischer (dernière consultation le 15 mai 2020).

2 Ulrich Heinemann, Die verdrängte Niederlage. Politische Öffentlichkeit und Kriegsschuldfrage in der Weimarer Republik, Göttingen, 1983; Wolfgang JäGER, Historische Forschung und politische Kultur in Deutschland. Die Debatte 1914-1918 über den Ausbruch des Ersten Weltkrieges, Göttingen, 1984; Imanuel GeIss, «Die manipulierte Kriegschuldfrage», Militärgeschichtliche Mitteilungen, 2 (1983), p. 31-60.

3 Michael Dreyer, Oliver Lembcke, Die deutsche Diskussion um die Kriegsschuldfrage 1918/19, Leipzig, 1993. 
population. S'appuyant sur une étude approfondie des faits et des sources, ces auteurs dissèquent sous un angle extrêmement critique toutes les tentatives des institutions, partis et associations pour nier la responsabilité allemande et pour dresser de véritables barrières de propagande contre une appréciation objective de la culpabilité de l'Empire allemand. Ils excluent ainsi totalement de l'analyse et de la critique historique une réflexion montrant que l'énergie extraordinaire déployée dans l'entre-deux-guerres pour protester de l'innocence allemande pouvait avoir été nourrie par la conviction de la grande majorité des Allemands que leur pays n'était aucunement le principal, voire l'unique responsable de la Première Guerre mondiale.

Cette approche très critique des historiens des années 1990 ne permet nullement de comprendre les gens de l'époque et passe complètement à côté de l'intensité du débat sur les responsabilités au lendemain du conflit mondial. Si ce dernier a bien fait l'objet d'une instrumentalisation politique massive, la raison principale en est que la question de la responsabilité de la guerre préoccupait vivement l'ensemble de la population et qu'elle est par conséquent inéluctablement entrée dans le viseur de la politique. Il suffit d'imaginer ce que ce reproche a dû signifier pour les Allemands, dont la plupart étaient intimement persuadés d'avoir mené une guerre de légitime défense, de se retrouver responsables «d'au moins sept millions de morts», qui, comme l'écrivit Georges Clemenceau à la délégation allemande à Versailles, "gisent enterrés en Europe » ${ }^{(4)}$.

Le regard (excessivement) critique de la recherche des années 1980 et 1990 est le résultat d'un consensus général né dans les années 1970 à propos de la responsabilité allemande dans le déclenchement de la Première Guerre mondiale. Si les thèses de Fritz Fischer sur cette question avaient fait scandale dans les années 1960, dix ans plus tard elles relevaient déjà de la culture générale, que ce soit au sein de la recherche historique ou des milieux cultivés de la société allemande. Quant à Das deutsche Kaiserreich (l'Empire allemand), l'ouvrage de Hans-Ulrich Wehler maintes fois réédité qui établit une continuité entre l'impérialisme insatiable de l'Allemagne et le déclenchement de la guerre, il est devenu un manuel à l'université comme au lycée ${ }^{(5)}$.

De cette conviction fondamentale de la responsabilité prédominante de l'Allemagne dans la Première Guerre mondiale découle le regard actuel empreint d'un préjugé négatif sur l'attitude des Allemands et tout particulièrement sur le comportement de la délégation allemande à Versailles à l'égard des traités de paix de 1919.

Eberhard Kolb, en 2005, a su défendre avec conviction l'idée selon laquelle les Allemands auraient pu obtenir de meilleures conditions de paix s'ils ne s'étaient pas polarisés sur la question de la responsabilité. Avec lui, la recherche a pris un nouveau tournant:

«La fonction de l'article 231 était d'entériner dans les traités la responsabilité juridique de l'Allemagne pour les dommages causés et donc de donner une assise légale au droit de réparation. Dans un premier temps, il s'abstenait ainsi de jeter un discrédit moral massif sur l'Allemagne.»

4 Voir, par exemple: archive.org/stream/traitdepaixent00cana/traitdepaixent00cana_djvu.txt. Également cité dans Gerd Krumeich, L’impensable défaite. L'Allemagne déchirée, 1918-1933, traduction de Josie Mély, Paris, Belin, 2019.

5 Hans-Ulrich Wehler, Das deutsche Kaiserreich 1871-1918, Göttingen, 1973. 
Ce n'est que sous l'effet des protestations passionnées des Allemands à la publication du traité que l'article 231 prit, selon E. Kolb, le caractère d'un verdict de responsabilité morale ${ }^{(6)}$.

Dans ce contexte, Les somnambules de Christopher Clark ont jeté un pavé dans la mare. En délestant amplement l'Empire allemand de sa responsabilité dans l'entrée en guerre, il a eu un effet dé-traumatisant, qui se reflète dans le succès considérable de l'ouvrage en Allemagne, dont la première édition s'est vendue à plus de 350000 exemplaires $^{(7)}$. Pour les sciences historiques, C. Clark a introduit la possibilité d'un véritable revirement, qui aujourd'hui semble progressivement s'établir. Pourtant, je me suis retrouvé bien seul lorsque, au zénith de l'engouement pour Les somnambules, j'ai publié un ouvrage qui montrait qu'au-delà de toute l'estime que l'on peut avoir pour son travail sur les sources et la force de son exposé, C. Clark juge de façon un peu trop clémente la gestion de la crise de 1912-1914 par l'Empire allemand. S'il me paraît évident que l'Allemagne n'est pas la seule à avoir alimenté la poudrière qui a explosé en juillet 1914, les dirigeants de l'Empire ont clairement allumé la mèche fournie par l'impérialisme paneuropéen ${ }^{(8)}$.

Au demeurant, je pense que la conviction de la grande majorité des Allemands d'avoir été «encerclés» et d'avoir mené une guerre de légitime défense doit être prise bien plus fortement en compte dans l'analyse et l'appréciation historiques que l'ancienne historiographie «critique» ne l'a fait ou n'a souhaité le faire. Celle-ci a toujours jugé essentiel de démontrer que l'Allemagne n'avait précisément pas été "encerclée», mais qu'elle s'était elle-même «exclue du cercle» des grandes puissances. Mais cette "auto-exclusion» n'exclut pas le fait que la plupart des Allemands de l'époque étaient persuadés de la mauvaise intention des autres grandes puissances européennes à leur égard. En argumentant ainsi, je porte simplement plus d'attention au contexte et j'apporte un approfondissement nécessaire, au sens d'une 'compréhension' (Dilthey) des représentations mentales de l'époque.

Cette perspective rigoureusement contextualisée permet également à long terme d'apprécier plus justement l'état d'esprit de l'époque et les actions menées dans les années postérieures à 1918 sous l'impulsion de l'indignation générale. Ce n'est pas une coïncidence, pas plus que le seul fait d'une propagande de l'État et des partis ou associations, si la mobilisation contre la responsabilité imputée à l'Allemagne a été si massive. À considérer, même partiellement, la profusion de livres, brochures et articles de journaux sur le sujet, force est de conclure qu'aucun autre thème n'a plus enfiévré les Allemands que cette question de la responsabilité de leur pays dans une guerre mondiale qui a coûté la vie à deux millions de leurs soldats et dont les pertes militaires globales d'au moins dix millions d'hommes étaient un ordre de grandeur pas même imaginable à l'époque.

6 Eberhard Kolb, Der Frieden von Versailles, Munich, 2005, p. 66.

7 Christopher CLARK, Les somnambules. Été 1914: comment l'Europe a marché vers la guerre, traduction de Marie-Anne de BÉru, Paris, Flammarion, 2013; voir également ma critique de cet ouvrage dans Gerd Krumeich, Le feu aux poudres. Qui a déclenché la guerre en 1914?, traduction de Claudine LAYre, Paris, Belin (Alpha), 2018, en part. p. 70-76, 117-118.

8 G. Krumeich, Le feu aux poudres (note 7). 
Un petit tour d'horizon des principaux titres visant à innocenter l'Allemagne permet de saisir l'intensité et l'ampleur de cette campagne et la manière dont elle a réussi, quinze ans durant, à dominer le marché du livre allemand en avançant sans cesse des arguments et de nouvelles justifications:

- Mensuel Die Kriegsschuldfrage (La question de la responsabilité de la guerre) de 1922 à $1943^{(9)}$;

- Documents iconographiques sur la question des responsabilités quant à l'éclatement de la guerre en 1914;

- Leitfaden zur Kriegsschuldfrage (Guide sur la question de la responsabilité de la guerre $)^{(10)}$ par le comte Max von Montgelas, coéditeur des Deutsche Dokumente zum Kriegsausbruch (Documents allemands sur le déclenchement de la guerre) et de l'anthologie de dossiers diplomatiques Die große Politik der Europäischen Kabinette (La grande politique des cabinets européens);

- Leitsätze zur Kriegsschuldfrage (Préceptes sur la question de la responsabilité de la guerre) du même auteur ${ }^{(11)}$;

- Grundzüge der Kriegsschuldfrage (Les grandes lignes de la question de la responsabilité de la guerre) ${ }^{(12)}$;

- Anklage und Widerlegung. Taschenbuch zur Kriegsschuldfrage (Accusation et réfutation. Brochure sur la question de la responsabilité de la guerre) et son appendice: Erklärungen der deutschen Regierungen zur Kriegsschuldfrage (Déclarations des gouvernements allemands sur la question de la responsabilité de la guerre), jusqu'en $1928^{(13)}$;

- Die Kriegsschuldfrage in der deutschen Schule (La question de la responsabilité de la guerre à l'école allemande) ${ }^{(14)}$;

- Die Kriegsschuldfrage. Ein Verzeichnis der Literatur des In- und Auslandes (La question de la responsabilité de la guerre. Inventaire de la littérature allemande et étrangère $)^{(15)}$;

- Die Lüge von der deutschen Kriegsschuld im Geschichtsunterricht der deutschen Schulen (Le mensonge de la responsabilité allemande dans les cours d'histoire des écoles allemandes) ${ }^{(16)}$.

9 Die Kriegsschuldfrage, sous la direction d'Alfred von WEgERER, Berlin, Zentralstelle für Erforschung der Kriegsursachen, Berliner Monatshefte für internationale Aufklärung.

10 Berlin, éditions de Gruyter, 1923.

11 Max Graf Montgelas, Leitsätze zur Kriegsschuldfrage, Berlin, de Gruyter, 1927.

12 Georg Karo, Grundzüge der Kriegsschuldfrage, Munich, Süddeutsche Monatshefte, 1926.

13 Anklage und Widerlegung. Taschenbuch zur Kriegsschuldfrage, sous la direction de Hans DrAEGER, Berlin, Arbeitsausschuß deutscher Verbände, sans date (1928).

14 Georg Hanke, Die Kriegsschuldfrage in der deutschen Schule, Langensalza, Julius Beltz, 1933.

15 Publié par le Börsenverein der deutschen Buchhändler (Association professionnelle allemande du Livre), Leipzig, 1925.

16 Richard Neumann, Die Lüge von der deutschen Kriegsschuld im Geschichtsunterricht, Langensalza, Beyer \& Söhne, 1924. 


\section{Réminiscence en 1933}

Toute l'ampleur de la question des responsabilités, si profondément émotionnelle pour les Allemands, se révèle sans doute le mieux à l'aune d'un incident qui a peu retenu l'attention à ce jour. Dans son discours du 23 mars 1933 plaidant pour l'obtention des pleins pouvoirs, Hitler déclara:

«Le succès de la révolution permit aux auteurs de l'attentat d'échapper à la justice. Cherchant une légitimation morale, ils affirmèrent que l'Allemagne, ou son gouvernement, portait la responsabilité du déclenchement de la guerre ${ }^{(17)}$.

S’il peut paraître étrange que dans une situation si décisive et entièrement tendue vers l'avenir Hitler ait mis l'accent sur la révolution de novembre 1918 et les débats sur la responsabilité, on sera encore plus surpris qu'Otto Wels, le chef du groupe socialiste au Reichstag, dans une réplique aussi courageuse que désespérée (et qui reste un modèle d'engagement démocratique dans des périodes désenchantées) lui ait répondu précisément sur ce point. Cela n’a pratiquement encore jamais été relevé, mais Wels y approuva aussi le programme de politique étrangère de Hitler. De plus, il souligna qu'il avait lui-même, dès février 1919, combattu la «contrevérité de la responsabilité de l'Allemagne dans le déclenchement de la guerre mondiale» à l'occasion d'un congrès socialiste à Berne. Et il abonda explicitement dans le sens de Hitler en renvoyant à «la folie des réparations».

Très agité, Hitler réagit sur-le-champ:

«Vous y venez tard, mais vous y venez! Vous déclarez que la social-démocratie approuve notre programme de politique étrangère, qu'elle rejette le mensonge de la responsabilité, qu'elle s'oppose aux réparations. Je ne poserai qu'une seule question: qu'en était-il de ce combat à l'époque où vous étiez au pouvoir en Allemagne? » ${ }^{(18)}$.

La dimension traumatique de la question des responsabilités pourrait-elle être plus éclatante que dans cette absurde joute oratoire livrée quelques instants avant la désintégration de la démocratie allemande?

\section{Les débuts du débat sur les responsabilités}

Mais revenons aux débuts du débat sur les responsabilités. Dès leur réponse à l'initiative de paix allemande de décembre 1916, les Alliés avaient mis en avant que le règlement des dommages provoqués par l’agression allemande de 1914 devait précéder toute conclusion de paix ${ }^{(19)}$. Cette exigence a été précisée et confortée dans l'échange de notes entre Wilson et le gouvernement allemand en novembre 1918. La réponse à la demande allemande de médiation rédigée et signée par le secrétaire d'État des ÉtatsUnis, Robert Lansing, aujourd'hui encore connue sous le nom de "note Lansing", indiquait:

«Les gouvernements alliés estiment qu'aucun doute n'est permis sur ce qu'implique cette disposition. Ils entendent par là que l'Allemagne indemnisera tous les dommages subis par

17 Cité d'après Max Domarus (dir.), Hitler. Reden 1932-1945, t. 1, Wiesbaden, 1973, p. 229.

18 Ibid.

19 Dreyer/Lembcke, Die deutsche Diskussion (note 3), p. 44-45. 
la population civile des Alliés et leurs biens en raison de l'agression allemande sur l'eau, sur terre et dans les airs ${ }^{(20)}$.

Toutes les réclamations de réparation se fondaient donc sur le postulat d'une "agression» allemande en juillet 1914, qui avait déclenché la guerre; raison pour laquelle l'Allemagne devait assumer la responsabilité de tous les dommages.

À l'époque, les Allemands ont traduit aggression par Angriff (attaque, offensive) ${ }^{(21)}$ et immédiatement souligné qu'ils étaient bien entendu disposés à payer pour les dommages résultant de leur offensive sur la Belgique et la France, mais pas pour ceux que les Alliés avaient eux-mêmes causés en détruisant des villages, villes et œuvres d'art, à l'image de la cathédrale de Laon. Ces derniers rejetèrent toutefois la distinction. Alors que Wilson avait promis une paix juste, les Allemands furent déçus, voire se sentirent trahis.

\section{4. À partir de novembre 1918, le scandale Eisner}

On comprendra aisément dans ces circonstances que Kurt Eisner, chef du parti d'extrême gauche, la USPD (Unabhängige Sozialistische Partei Deutschlands - Parti socialiste indépendant d'Allemagne), ministre-président de Bavière, ait déclenché un tollé lorsqu'il publia, quelques jours après sa prise de pouvoir révolutionnaire le 8 novembre 1918, une version - tronquée! - d'un rapport explosif du comte Lerchenfeld, ministre plénipotentiaire bavarois à Berlin, daté du 28 juillet 1914. En effet, ce rapport prouvait avec moult détails que le gouvernement allemand n'avait nullement essayé de tempérer l'intervention de l'Autriche-Hongrie contre la Serbie en juillet 1914. Bien au contraire, il avait conforté la monarchie habsbourgeoise dans son projet d'exploiter l'attentat de Sarajevo pour triompher définitivement de la Serbie.

Qu'a modifié exactement Eisner dans le «rapport Lerchenfeld»? La reproduction synchronoptique dans l'édition de Pius Dirr ${ }^{(22)}$ révèle qu'il n'a rien ajouté. Il a en revanche supprimé un long passage et intercalé quelques résumés. Pour mettre en exergue le sens de mots ou pans de phrases qui lui paraissaient particulièrement scandaleux, Eisner en a également augmenté l'interlettrage. Il a en outre laissé de côté le long développement montrant que les dirigeants berlinois pensaient que le conflit se limiterait certainement à l'Autriche-Hongrie et à la Serbie, c'est-à-dire qu'il serait possible de le «localiser», pour reprendre le terme si souvent brandi pendant la crise de juillet. Une seule chose importait à Eisner: le rapport prouvait que l'Allemagne était décidée à soutenir son alliée, même si les autres grandes puissances intervenaient, même au prix d'une guerre européenne. Il citait également certains détails en jouant sur l'interlettrage quand ceux-ci lui semblaient prouver que le gouvernement

20 "The Allied Governments feel that no doubt ought to be allowed to exist as to what this provision implies. By it they understand that compensation will be made by Germany for all damage done to the civilian population of the Allies and their property by the aggression of Germany, by land, by sea, and from the air", dans Materialien betreffend die Friedensverhandlungen, part. 1, Charlottenburg, 1919 , p. 6, souligné par l'auteur de l'article.

21 L'édition de sources, précieuse à maints égards, de Klaus ScHwABE, Quellen zum Friedensschluß von Versailles, Darmstadt, 1997, omet cette distinction, voir p. 68.

22 Pius Dirr (dir.), Bayerische Dokumente zum Kriegsausbruch und zum Versailler Schuldspruch, Munich/Berlin, 1925 (3éd.), p. 6 sq. 
allemand avait des intentions explicitement agressives et qu'il était résolu à en venir à une guerre générale ${ }^{(23)}$.

À parcourir les recherches menées sur la crise de juillet 1914 depuis les années 1960, on constate rapidement que les interprétations des historiens continuent à se diviser sur ce point. La prétention de «localiser» le conflit constituait-elle une preuve des visées fondamentalement pacifiques du gouvernement allemand ou était-elle l'arbre qui cachait la forêt d'une stratégie belliqueuse déjà arrêtée? Il n'est pas nécessaire de trancher ici cette question ${ }^{(24)}$. Mais la pérennité de la controverse indique qu'Eisner a certainement agi de bonne foi. Ainsi que nombre d'historiens aujourd'hui encore, il considérait l'intention de circonscrire le conflit à l'échelle régionale ( localisation») comme parfaitement secondaire, et il était tellement persuadé de la responsabilité unique de l'Allemagne dans le déclenchement de la guerre qu'il qualifia de «considérations quelconques", voire de «verbiage» tout ce qui, dans le «rapport Lerchenfeld», était susceptible de contredire sa thèse. Il attaquait de front l'argument principal des défenseurs de l'Allemagne, qui avaient toujours mis en avant l'intention du gouvernement allemand de limiter la guerre à l'Autriche-Hongrie et à la Serbie; cela prouvait que l'Allemagne n'avait jamais cherché à déclencher une guerre européenne entre les grandes puissances. Or, c'était précisément ce que lui reprochèrent les Alliés en amont du congrès de Versailles, ce qu'ils formulèrent de manière archétypique dans la «note de couverture» sur les conditions de paix.

Dans les rassemblements politiques où il prenait la parole, Eisner ne manquait jamais de proclamer la «responsabilité unilatérale» de l'Allemagne. Lors de la «conférence du Reich» des Conseils d'ouvriers et de soldats organisée le 25 novembre 1918, il échangea de vifs propos avec Philipp Scheidemann, président du MSPD (Mehrheitssozialdemokratische Partei Deutschlands - Parti social-démocrate majoritaire d'Allemagne) et, depuis le 9 novembre 1918, membre du gouvernement révolutionnaire, le Rat der Volksbeauftragten (Conseil des délégués du peuple). Scheidemann accusa Eisner d'avoir asséné un "coup de massue» à son pays en publiant les documents des archives bavaroises sur la crise de juillet. Eisner, qui utilisa cette opportunité (et bien d'autres) pour régler ses comptes avec les dirigeants du MSPD, exigea qu'on arrête les hommes politiques qui, à l'image de Jagow, Zimmermann et Erzberger, avaient poussé l'Allemagne dans le conflit et avaient exigé des buts de guerre considérables. À ses yeux en effet, ils avaient agi en «criminels» irresponsables, aussi bien avant que pendant ce conflit.

Dès le 27 novembre 1918, Bethmann Hollweg, l'ancien chancelier du Reich et acteur décisif de la crise de juillet, prit longuement position sur ces documents dans le quotidien Deutsche Allgemeine Zeitung. Il était exact, affirma-t-il, qu'on avait accepté en juillet 1914 «des complications guerrières ultérieures» pour sauver l'alliance avec l'Autriche-Hongrie, seul appui de l'Allemagne. Certainement aussi, poursuivait Hollweg, l'ultimatum de l'Autriche-Hongrie à la Serbie avait été trop brutal. Mais jamais le gouvernement allemand n'avait planifié de déclencher une guerre mondiale. Le paramètre décisif avait été la mobilisation générale en Russie. Commentant un article du

23 Pour plus de développements sur la polémique déclenchée par les publications d'Eisner, voir G. KRUMEICH, L'impensable défaite (note 4), p. 135 à 141.

24 J'ai longuement discuté la problématique de la localisation dans Le feu aux poudres (note 7), p. 89-116. 
pacifiste Friedrich W. Foerster qui minimisait les activités d'Eisner, Hans Delbrück, historien et critique impitoyable du militarisme prussien, déclara pour sa part: «La publication d'Eisner est l'une des falsifications diplomatiques les plus impudentes et infâmes de l'histoire mondiale. Que le professeur Foerster puisse considérer excuser un tel acte témoigne d'une insensibilité morale dont je ne l'aurais pas cru capable» ${ }^{(25)}$.

\section{Le poids de la question de la responsabilité pour les négociations de Versailles}

Comme évoqué plus haut, la plupart des chercheurs allemands partent aujourd'hui du postulat selon lequel le comportement si impitoyable des Alliés à l'égard de l'Allemagne s'explique par l'attitude de la délégation allemande à Versailles, en premier lieu par la manière inconvenante dont son chef, le comte Brockdorff-Rantzau, a accueilli les propositions de paix de l'Entente ${ }^{(26)}$. Or, je contredis résolument cette thèse au vu des principales prises de position des Alliés avant même le début des «négociations » avec les Allemands. Ainsi, le président de la République française, Raymond Poincaré, s'exprimat-il en ces termes lors de l'ouverture des négociations versaillaises le 18 janvier 1919:

«Ce qui vous donne toute qualité pour établir une paix de justice, c'est qu'aucun des peuples dont vous êtes les mandataires n'a trempé dans l'injustice. L'humanité peut vous faire confiance, parce que vous n'êtes pas de ceux qui ont violé les droits de l'humanité [...] La vérité, toute couverte de sang, s'est déjà évadée des archives impériales. La préméditation du guet-apens est aujourd'hui clairement démontrée » ${ }^{(27)}$.

De même, le rapport présenté le 28 mars 1919 par la «Commission des responsabilités» des Alliés évoque une «intention préméditée» de l'Allemagne (et de l'AutricheHongrie) de déclencher une guerre. Pour le prouver, le rapport passe en revue la crise de juillet 1914 presque jour après jour et conclut:

«La guerre a été préméditée par les puissances centrales ainsi que par leurs alliées, la Turquie et la Bulgarie, elle est le résultat d'actes délibérément commis dans l'intention de la rendre inévitable.

L'Allemagne, d'accord avec l'Autriche-Hongrie, a travaillé délibérément à faire écarter les nombreuses propositions conciliatrices des puissances de l'Entente et à réduire à néant leurs efforts répétés pour éviter la guerre» ${ }^{(28)}$.

La déclaration que Brockdorff-Rantzau lut (assis!) le 7 mai 1919 après avoir reçu les conditions de paix n'est donc en rien la provocation éhontée que les chercheurs allemands ont voulu y voir depuis, mais une réponse très pondérée aux accusations évoquées ci-dessus - et à bien d'autres - des vainqueurs.

"On exige de nous que nous nous reconnaissions comme les seuls coupables de la guerre; un tel aveu dans ma bouche serait un mensonge. Loin de nous l'idée de vouloir rejeter toute responsabilité de l'Allemagne dans l'avènement de cette guerre mondiale et dans la

25 Toutes les prises de position citées ici sont extraites de P. DirR, Bayerische Dokumente (note 22), p. 56 sq.; Max Weber le grand sociologue allemand a également pris clairement position contre Eisner, voir à ce propos G. KRUMEICH, L’impensable défaite (note 4), p. 158-159.

26 E. Kolb, Versailles (note 6); sur la même ligne Jörn Leonhard, Der überforderte Frieden. Versailles und die Welt 1918-1923, Munich, 2018, p. 963 sq.; Eckart Conze, Die große Illusion, Versailles 1919 und die Neuordnung der Welt, Munich, 2018, p. 360 sq.

27 Raymond Poincaré, Messages, discours, allocutions, t. 2, Paris, 1920, p. 132-133.

28 Citation dans Albert de Geouffre de Lapradelle (dir.), La paix de Versailles, t. 1, Paris, 1920 , p. 87. 
manière dont elle a été conduite. [...] Mais nous refusons expressément que l’Allemagne, dont le peuple était convaincu de mener une guerre défensive, doive en endosser seule la responsabilité» ${ }^{(29)}$.

\section{Les publications allemandes officielles}

Dès la fin 1918, le dirigeant socialiste Karl Kautsky fut chargé par le gouvernement révolutionnaire, le Rat der Volksbeauftragten, de remettre une documentation sur la crise de juillet 1914 au ministère des Affaires étrangères avant le mois de mars 1919. Kautsky remplit sa mission dans les temps impartis. En revanche, la publication du rapport fut ajournée, car, à l'étude des dossiers des Affaires étrangères, concernant la crise de juillet 1914, Kautsky avait acquis la conviction que le gouvernement allemand avait une responsabilité massive, si ce n’est décisive, dans le déclenchement du conflit. La documentation parut fin 1919 sous la direction de personnalités dont l'intégrité et le pacifisme étaient internationalement reconnus, le comte Max Montgelas et Walther Schücking. Kautsky avait été « débarqué» en raison de sa position critique à l'égard de la politique allemande dans la crise de juillet, mais avait donné son aval aux formulations mesurées, voire légèrement apologétiques de l'introduction. Toutefois, il n'était manifestement pas satisfait et, avant même la sortie de ce document, il fit paraître une brochure intitulée Comment s'est déclenchée la guerre mondiale. Dans la préface, Kautsky déplorait amèrement l'absence de soutien officiel du gouvernement dans ses recherches puis, en près de 200 pages, il déroulait la préhistoire plus ou moins récente du conflit mondial, telle que les sources examinées par ses soins la présentaient. Il condamnait impitoyablement les gouvernements allemand et autrichien et n'hésitait pas à parler de «complot» fomenté par les deux autorités contre la paix en Europe. Le pamphlet de Kautsky provoqua des discussions enflammées. Hans Delbrück, critique implacable du militarisme wilhelminien et à la tête de la commission d'enquête de l'Assemblée nationale sur la question de la responsabilité, lui reprocha de se laisser bien trop emporter par ses préjugés à l'égard des dirigeants de 1914. Kautsky aurait «grossièrement bafoué les premiers devoirs de l'historien, à savoir restituer les faits de façon correcte et minutieuse, identifier les motifs des acteurs, saisir leur pensée et exposer ces dernières avec exactitude ${ }^{(30)}$.

À l'occasion du «Dixième anniversaire de l'entrée en guerre», le gouvernement allemand publia le 29 août 1924 une déclaration officielle, dans laquelle il affirmait:

«La conclusion selon laquelle l’Allemagne a déclenché la guerre mondiale par son offensive, et qui nous a été imposée par le traité de Versailles avec une violence inouie, contredit les faits historiques. Le gouvernement du Reich déclare donc ne pas l'accepter. Le peuple allemand exige à bon droit d'être délesté du fardeau de cette accusation erronée. Tant que cela n'est pas le cas et qu'un membre de la communauté internationale est taxé de crimes contre l'humanité, il est impossible de parachever une entente véritable pas plus que la réconciliation entre les peuples» ${ }^{(31)}$.

29 Cité d'après K. Schwabe, Quellen zum Friedensschluß (note 21), p. 243.

30 Hans Delbrück, Kautsky und Harden, Berlin, 1920, p. 6.

31 Herbert Michaelis et Ernst Schraepler (dir.), Ursachen und Folgen. Vom deutschen Zusammenbruch 1918 und 1945 bis zur staatlichen Neuordnung Deutschlands in der Gegenwart, t. 6, Berlin, Dokumenten-Verlag Dr. Herbert Wendler (s. d.), p. 122. 
Puis, dix ans après la signature du traité de Versailles, le président de la République et le gouvernement allemand proclamèrent dans une déclaration commune:

«Aujourd'hui est un jour de deuil. Dix années se sont écoulées depuis que les négociateurs allemands de la paix ont été forcés à Versailles d'apposer leur signature en bas d'un document qui signifiait une amère déception pour tous les sympathisants du droit et d'une paix authentique. [...] L'Allemagne a signé le traité sans toutefois reconnaître que le peuple allemand était l'instigateur de cette guerre. Ce reproche hante notre peuple et ébranle la confiance entre les nations. Nous nous savons unis à tous les Allemands pour rejeter l'affirmation de la responsabilité unilatérale de l'Allemagne dans la guerre » ${ }^{(32)}$.

Le rejet de la responsabilité est donc directement corrélé à la lutte contre «Versailles» dans sa globalité, ce qui explique pourquoi la question des responsabilités dans le déclenchement de la catastrophe européenne n'a jamais fait l'objet d'un débat nuancé en Allemagne. Hormis la position très marginale d'Eisner et de ses alliés de gauche, tous les partis et toutes les associations du centre, de la droite et de la gauche modérée s'entendirent sur un rejet général et quasi unanime de l'idée de la culpabilité unilatérale de l'Allemagne.

\section{Les responsabilités et la légende du coup de poignard}

Ce consensus fondamental n'a toutefois pas donné lieu à une alliance ralliant la totalité de l'échiquier politique contre les allégations et revendications de l'étranger, de la France en particulier. Il faut en chercher la raison dans le fait que ce débat sur la responsabilité était indissolublement entremêlé au reproche du coup de poignard. Pour la droite en effet, il restait irrévocable que Versailles et l'accusation de la responsabilité n'auraient jamais vu le jour si la révolution n’avait pas éclaté. Les propos, cités plus haut, de Hitler en mars 1933 reprenaient un axiome du discours politique de la droite tout au long de la République de Weimar. Le grief du coup de poignard a toutefois connu deux variantes, régulièrement invoquées dans les longs débats de la commission d'enquête de l'Assemblée nationale, puis d'une commission analogue instituée par le Reichstag (Parlement):

a) on aurait pu tenir un peu plus longtemps et obtenir de meilleures conditions de paix;

b) les conspirateurs juifs et communistes ont empêché la victoire de l'armée allemande, dont les assauts étaient pourtant victorieux.

Soulignons ici que la version modérée, aujourd'hui malheureusement quasi occultée dans les essais traitant du coup de poignard, était alors bien plus populaire au sein de la commission d'enquête et de la sphère politique que la variante extrémiste, qui n'a d'abord été défendue que par les nazis et autres associations völkisch ${ }^{(33)}$ ou quelques

32 Ibid., t. 9, p. 39.

33 «Le mouvement völkisch, qui prend corps par étapes à la charnière des $\mathrm{XIX}^{\mathrm{e}}$ et $\mathrm{XX}^{\mathrm{e}}$ siècles [... est un] mouvement de quête et de protestation à caractère hétérogène et nationaliste radical [...] Qu'il s'agisse de la pensée ou du mouvement, l'adjectif völkisch est [...] foncièrement défini par le critère de la "race", le concept de race oscillant entre une définition biologique et une définition spiritualiste [...] Elle part du postulat que les Aryens [...] forment la race supérieure parmi toutes les races humaines [...] À cette conviction d'une supériorité de la race répond dans l'idéologie völkisch [...] l'idée d'une menace existentielle provenant d'autres races classées comme inférieures, ce qui permet de légitimer 
rares militaires. Pourtant, on ne connaît aujourd'hui pratiquement plus que cette dernière version, parce qu'elle est restée la seule valide avec la «prise de pouvoir» d'Adolf Hitler puis tout au long des années 1930.

La première variante reste aujourd'hui une problématique que la plupart des chercheurs évitent soigneusement. Elle mériterait pourtant d'être discutée dans une perspective historique et historienne ${ }^{(34)}$. Tout l'enjeu est de déterminer si la révolution a scellé l'armistice dans la forme que l'on connaît, c'est-à-dire la capitulation inconditionnelle à peine déguisée de l'Allemagne. La grande majorité des historiens allemands d'aujourd'hui pense que l'armée était tout simplement défaite en novembre 1918 et qu'il aurait été impossible ou insensé de persévérer ${ }^{(35)}$. En conséquence, la révolution n'a rien à voir avec la débâcle et tous les travaux actuels sur la révolution ignorent d'ailleurs largement la capitulation simultanée de l'armée allemande.

Il est intéressant de relever que la recherche française sur ce thème, peu connue en Allemagne, porte un regard différent sur la situation. Pour le $90^{\mathrm{e}}$ anniversaire de la fin de la guerre en 2008, les archives militaires françaises ont publié un catalogue d'exposition richement illustré sur L'étrange victoire de $1918^{(36)}$. S'appuyant sur une quantité considérable de sources de l'époque, il révèle qu'en octobre-novembre 1918, la direction militaire de l'Entente ne comptait guère sur une issue prochaine du conflit. Les commandants en chef des armées alliées - Haig, Joffre, Foch - prévoyaient des opérations pour le début de l'année 1919 qui, espérait-on, permettraient enfin de briser la résistance allemande. Si, fin octobre-début novembre 1918, la totalité des armées allemandes avait reculé, elles tenaient pourtant fermement leurs positions. Au demeurant, la motivation au combat, très chancelante depuis juillet 1918, avait encore notablement baissé sous l'effet de la demande allemande de cessez-le-feu de la fin septembre 1918. Des recherches récentes ont établi que les désertions massives, les redditions de prisonniers et la montée en flèche de l'indiscipline ont débuté seulement après la demande de cessez-le-feu faite par Ludendorff ${ }^{(37)}$. De son côté, la révolution n'est évidemment pas sans rapport avec cette dernière, puisqu'elle était à l'origine une «révolution des soldats»: une réaction à la "re-mobilisation», et aux opérations de pur prestige, à l'image de la stratégie de la canonnière planifiée dans la rade de Wilhelmshaven et empêchée par la mutinerie des matelots. Dans une certaine mesure, la révolution munichoise du 8 novembre, lancée par Eisner, puise à cette même eau. Partie d'une manifestation contre les projets et mesures de mobilisation des hommes

le militarisme et le bellicisme» (Uwe Puschner, «La pensée völkisch», Dictionnaire historique et critique du racisme, Paris, PUF «Quadrige», 2013, p. 1874-1875, NDT).

34 Je prépare actuellement une monographie sur cette (si épineuse) question.

35 Pour des détails voir G. Krumeich, L’impensable défaite (note 4), p. 93-103.

36 Frédéric Guelton (dir.), L'étrange victoire. Archives du ministère de la Guerre et témoignages inédits, Paris, 2008.

37 Christoph JAHR, «Bei einer geschlagenen Armee ist der Klügste, wer zuerst davonläuft. Das Problem der Desertion im deutschen und britischen Heer 1918 », in: Jörg Duppler, Gerhard P. Gross (dir.), Kriegsende 1918. Ereignis, Wirkung, Nachwirkung, Munich, 1999, p. 241-272; David Stevenson, With our Backs to the Wall. Victory and Defeat in 1918, Cambridge, MA, 2011; Scott Stephenson, The Final Battles: Soldiers of the Western Front and the German Revolution, Cambridge, 2009; Alexander Watson, Enduring the Great War: Combat, Morale and Collapse in the German and British Armies 1914-1918, Cambridge, 2008. 
« exemptés », elle s'est muée en rassemblement de masse grâce à la participation de ces derniers et des soldats, pour se poursuivre par l'occupation des postes de commande politiques et militaires de la capitale bavaroise.

C'est exactement le contexte dans lequel s'inscrivaient les propos de Hitler en 1933, lorsqu'il affirma que la révolution de novembre avait donné le "coup de poignard» décisif dans le dos de l'armée allemande. Il convient, sur le plan heuristique et historique, de dissocier strictement les conclusions délirantes de Hitler et de ses affidés - à savoir que les «Juifs et bolcheviques" auraient intentionnellement porté ce coup pour anéantir l'Allemagne - de la contribution indubitable de la révolution sinon à la défaite, du moins à la forme de capitulation qui l'a suivie. Il est hautement probable qu'Erzberger aurait bénéficié à Compiègne d'une base de négociations plus favorable face à Foch s'il avait pu, au moins, menacer de rompre les pourparlers. Car rien ne permet d'affirmer que les Français et leurs alliés auraient été en mesure de remobiliser leurs soldats pour une offensive en Allemagne si ces derniers, également plus que las de la guerre, avaient su que l'enjeu de la bataille ne résidait pas dans la victoire, mais dans des vœux d'expansion et des exigences démesurées de leurs chefs d'État et d'armée ${ }^{(38)}$. En cas de poursuite des combats, Erzberger n'aurait certainement pas été contraint de reconnaître que "l'agression » de l'Allemagne en 1914 la rendait responsable de tous les dommages de guerre subis par les États de l'Entente et leurs citoyens ni qu'elle devait en conséquence payer leur réparation; prémisse qui était non seulement entérinée par l'article sur la « responsabilité de la guerre» du traité de Versailles, mais faisait déjà partie intégrante des clauses de l'armistice du 11 novembre 1918. Erzberger ne l'avait signé qu’après avoir été prévenu par l'état-major militaire qu'il devait, coûte que coûte, accepter l'armistice sans condition, car la révolution interdisait toute poursuite des combats ${ }^{(39)}$.

\section{Conclusion}

Ainsi la défaite de 1918 est-elle restée la plaie ouverte du corps de la république - une blessure qui n'a pu guérir d'aucune manière jusqu'en 1933. Les promesses et les actions concrètes de Hitler en faveur de la révision de Versailles - notamment pour révoquer en 1934 l'article 231 du traité de 1919 - lui ont apporté le crédit dont il avait besoin pour faire accepter son régime de non-droit à des millions d'Allemands, qui ne se reconnaissaient cependant pas dans son extrémisme.

\section{Traduction de Valentine Meunier, relecture de Martine Sgard}

38 Voir Bruno Cabanes, La victoire endeuillée. La sortie de guerre des soldats français (1918-1920), Paris, 2004; il n'est ainsi pas fortuit que les soldats envoyés sur le front de l'Est après la signature de l'armistice en vue de combattre le communisme ne fussent pas des appelés, mais essentiellement des militaires de carrière. Jean-Jacques Becker et Gerd Krumeich, La Grande Guerre. Une histoire franco-allemande, Paris, Tallandier, 2008, p. 299 sq.

39 Amtliche Urkunden zur Vorgeschichte des Waffenstillstandes 1918, Berlin, 1928 (4éd.), nº 107, p. 265. 


\section{Résumé}

Cet article s'essaie à une réinterprétation du débat sur les "responsabilités " du déclenchement de la guerre mondiale, avant, pendant et après les négociations du traité de Versailles. Il traite en particulier de l'affirmation selon laquelle les Allemands étaient "seuls responsables" du déclenchement de la guerre et du rejet global de cette accusation par les Allemands. Cependant, ces derniers ne purent s'entendre sur une action commune pour lutter contre "Versailles", car la droite accusait la gauche d'être responsable de la défaite et donc du traité. L'article cherche à montrer que l'accusation du "coup de poignard dans le dos" n'était pas entièrement dénuée de fondement. Mais elle perdit toute vraisemblance lorsque la droite s'en empara et prétendit que l'Allemagne avait été délibérément dépossédée de sa victoire par les juifs et les communistes.

\section{Zusammenfassung}

Dieser Aufsatz versucht eine Neuinterpretation der Debatte um die „Verantwortlichkeiten" für den Ausbruch des Weltkrieges, vor, während und nach den Versailler Vertragsverhandlungen. Es geht insbesondere um die Behauptung einer „Alleinschuld“ der Deutschen für den Ausbruch des Krieges und die ganz allgemein geteilte Abwehr dieses Vorwurfs durch die Deutschen. Diese konnten sich aber im Kampf gegen „Versailles" nicht auf gemeinsame Aktion verständigen, weil die Rechte die Linke anklagte, durch den "Dolchstoß" schuld zu sein an der Niederlage, welche erst „Versailles“ möglich gemacht hatte. In diesem Beitrag wird versucht, zu zeigen, dass der „Dolchstoß“Vorwurf einen realistischen Kern hatte, dass aber durch die falsche Behauptung der Rechten, dass Deutschland durch die Juden und Kommunisten bewusst um den Sieg gebracht worden war, „totalisiert“ und damit zutiefst unglaubwürdig wurde. 